\title{
The Flavin Reductase MsuE Is a Novel Nitroreductase that Can Efficiently Activate Two Promising Next-Generation Prodrugs for Gene-Directed Enzyme Prodrug Therapy
}

Laura K. Green ${ }^{1}$, Mathew A. Storey ${ }^{1}$, Elsie M. Williams ${ }^{1,2}$, Adam V. Patterson ${ }^{3,4}$, Jeff B. Smaill ${ }^{3,4}$, Janine N. Copp ${ }^{1,2,3}$ and David F. Ackerley ${ }^{1,2,3, *}$

1 School of Biological Sciences, Victoria University of Wellington, Kelburn Parade, Wellington 6140, New Zealand

2 Victoria University Centre for Biodiscovery, School of Biological Sciences, Victoria University of Wellington, Wellington 6140, New Zealand

3 Maurice Wilkins Centre for Molecular Biodiscovery, School of Biological Sciences, University of Auckland, Auckland 1142, New Zealand

4 Auckland Cancer Society Research Centre, University of Auckland, Grafton, Auckland 1142,

New Zealand

* Author to whom correspondence should be addressed; E-Mail: david.ackerley@vuw.ac.nz;

Tel.: +64-4-463-5576; Fax: +64-4-463-5331.

Received: 31 May 2013; in revised form: 23 July 2013 / Accepted: 26 July 2013 /

Published: 8 August 2013

\begin{abstract}
Bacterial nitroreductase enzymes that can efficiently catalyse the oxygenindependent reduction of prodrugs originally developed to target tumour hypoxia offer great potential for expanding the therapeutic range of these molecules to aerobic tumour regions, via the emerging cancer strategy of gene-directed enzyme prodrug therapy (GDEPT). Two promising hypoxia prodrugs for GDEPT are the dinitrobenzamide mustard PR-104A, and the nitrochloromethylbenzindoline prodrug nitro-CBI-DEI. We describe here use of a nitro-quenched fluorogenic probe to identify MsuE from Pseudomonas aeruginosa as a novel nitroreductase candidate for GDEPT. In SOS and bacteria-delivered enzyme prodrug cytotoxicity assays MsuE was less effective at activating CB1954 (a first-generation GDEPT prodrug) than the "gold standard" nitroreductases NfsA and NfsB from Escherichia coli. However, MsuE exhibited comparable levels of activity with PR-104A and nitro-CBI-DEI, and is the first nitroreductase outside of the NfsA and NfsB enzyme families to do so. These in vitro findings suggest that MsuE is worthy of further evaluation in in vivo models of GDEPT.
\end{abstract}


Keywords: gene therapy; GDEPT; nitroaromatic prodrug; nitroreductase; CB1954; PR-104A; Nitro-CBI-DEI; SOS chromotest

\section{Introduction}

In gene-directed enzyme prodrug therapy (GDEPT), tumour tropic vectors are "armed" with a transgene that encodes a prodrug converting enzyme, enabling them to selectively sensitise tumour cells to that prodrug. A historical limitation of cancer gene therapies is their inability to transfect more than a small minority of cancerous cells [1,2]. In GDEPT, low transfection rates can be countered to a substantial degree by: (I) using a prodrug that exerts a strong bystander effect (i.e., the active metabolite(s) can efficiently penetrate and kill untransfected cells proximal to the site of activation); and (II) developing more efficient enzymes to achieve maximal prodrug conversion.

Therapeutic genes employed in GDEPT are typically non-human in origin, to minimise the potential for prodrug activation by endogenous enzymes in healthy tissue. Two of the most widely studied genes for GDEPT are herpes simplex virus thymidine kinase, which was evaluated in phase III clinical trials in combination with the nucleoside analogue prodrug ganciclovir [3]; and cytosine deaminase from Escherichia coli, which has undergone phase I trial in combination with another nucleoside analogue prodrug, 5-fluorocytosine [4]. These systems enjoy the substantial benefit that their prodrugs have each achieved independent clinical utility; but they also suffer from the limitations that only actively dividing cells are targeted, that their activated metabolites inhibit replication of viral vectors, and their cell-to-cell bystander effects are enhanced by functional gap junctions, which many tumour cells lack $[1,2,5]$.

A third enzyme-prodrug combination that has undergone clinical evaluation for GDEPT is E. coli NfsB (NfsB_Ec) in combination with CB1954 [5-(aziridin-1-yl)-2,4-dinitrobenzamide] [6,7]. The simultaneous 2-electron reduction of CB1954 by NfsB_Ec generates activated metabolites that can freely diffuse across cell membranes, forming adducts and DNA crosslinks, and inducing apoptosis in both replicating and quiescent tumour cells [8,9]. Nonetheless, results of the clinical trials were somewhat equivocal — while there was evidence for a slight anti-tumour effect, CB1954 was also discovered to exhibit dose-limiting hepatotoxicity, and the administered dose yielded a serum concentration of prodrug that was approximately two orders of magnitude lower than the $K_{m}$ of NfsB_Ec [6,7,10]. In our on-going research we have sought to improve the efficacy of CB1954 GDEPT through identification and engineering of nitroreductases that exhibit superior activity over NfsB_Ec at concentrations of prodrug that are attainable in vivo [11-14]. However, it is also known that CB1954 exhibits a relatively modest bystander effect relative to other nitroaromatic prodrugs with potential utility in GDEPT [15]. Thus, we have sought in parallel to identify superior prodrug substrates for nitroreductase GDEPT.

Two particularly promising next-generation prodrugs for nitroreductase GDEPT are nitro-CBI-DEI (nitro-CBI-5-[(dimethylamino)ethoxy]indole [16,17]) and PR-104A (2-(2-bromoethyl)-2-\{[(2-hydroxyethyl) amino]carbonyl -4,6-dinitroanilino)ethyl methanesulfonate [18]), each originally developed to be activated by tumour hypoxia. The rationale underpinning design of these nitro-triggered hypoxia prodrugs is that human oxidoreductases reduce these molecules via single-electron transfer, forming an initial nitro radical anion that molecular oxygen in healthy tissue rapidly back-oxidises, restoring the prodrug form 
in a "futile cycle" [19]. In contrast, we have shown that multiple bacterial nitroreductases from different enzyme families have the ability to reduce these molecules via oxygen-independent concerted two electron steps, yielding end metabolites that are highly cytotoxic and also exhibit substantial bystander effects $[14,17]$.

Previously, we have identified five different oxygen-independent bacterial enzyme families (NfsA, $\mathrm{NfsB}, \mathrm{NemA}$, AzoR and MdaB, each named after the orthologous enzyme from E. coli) as containing nitroreductases that can activate at least one of CB1954, PR-104A and nitro-CBI-DEI [14,17]. Of these, $\mathrm{NfsA}$ and NfsB family members consistently exhibit substantially greater activity with all three prodrugs than any of the other nitroreductase families. Two other enzyme families have been identified as having CB1954 reductase activity, YieF [20] and YwrO [9,21]; however to date we have not found any members of these families to exhibit measurable levels of activity with either PR-104A or nitro-CBI-DEI. Here we describe identification of MsuE from Pseudomonas aeruginosa as a novel nitroreductase, the first report of a non-NfsA or NfsB type enzyme to exhibit comparable levels of activity with these next-generation prodrugs for GDEPT.

\section{Results and Discussion}

\subsection{Identification of P. aeruginosa MsuE as a Nitroreductase Enzyme}

In our ongoing attempts to identify the most promising nitroreductases for GDEPT we have conducted a systematic evaluation of $\mathrm{Nfs} A, \mathrm{NfsB}, \mathrm{NemA}$, AzoR and MdaB family members from (to date) 25 different bacterial species [14,22]. We have augmented this family-based approach with individual evaluations of unrelated candidates, based on published structural and functional characteristics consistent with possible nitroreductase activity (i.e., homodimeric, NAD(P)H-consuming, flavin-cofactordependent, cytoplasmic oxidoreductases [14,23]). One such candidate was MsuE from P. aeruginosa, previously characterised as an NADH-dependent flavin mononucleotide (FMN) reductase that provides reduced FMN to power desulfonation of alkanesulfonates [24,25]. In an initial rapid qualitative test for nitroreductase activity, employing the nitro-quenched fluorophore FSL 61 as a generic nitroreductase substrate (as per [14]), a nitroreductase gene knockout strain of E. coli over-expressing MsuE from plasmid pUCX exhibited comparable levels of fluorescence to isogenic strains over-expressing E. coli NfsA (NfsA_Ec) or NfsB_Ec (Figure 1). In contrast, an empty-plasmid control strain was unable to activate the fluorophore (Figure 1). SDS-PAGE analysis coupled with scanning densitometry indicated that MsuE was expressed from pUCX in E. coli at approximately 35\% the level of NfsA_Ec and 38\% the level of NfsB_Ec.

\subsection{MsuE Is Genetically Distinct from Previously Identified Bacterial Nitroreductases}

When aligned against members of all other previously identified bacterial nitroreductase families using ClustalW2, with an out-group of human $\mathrm{NAD}(\mathrm{P}) \mathrm{H}$ quinone oxidoreductase 1 (NQO1, also known as DT-diaphorase [26]), the $m s u E$ gene formed a distinct clade (Figure 2). At an amino acid level, MsuE and its E. coli orthologue SsuE share nearly 30\% identity with one other, but each less than $15 \%$ identity with members of the AzoR family, their nearest neighbours in the ClustalW2 alignment. These observations suggest that MsuE belongs to a separate enzyme family from all other 
previously identified nitroreductases. Of particular note, our alignments revealed two highly conserved regions in each of the NfsA and NfsB families (NfsA: AAES[L/M/Q]G[F/L]GxxIG[A/G][I/L/M/V]R and $\mathrm{KPR}[\mathrm{L} / \mathrm{M}] \mathrm{Pxx}[\mathrm{A} / \mathrm{L} / \mathrm{M} / \mathrm{V}][\mathrm{I} / \mathrm{L} / \mathrm{M} / \mathrm{V}] \mathrm{xHE}[\mathrm{E} / \mathrm{N}]$; and $\mathrm{NfsB}:[\mathrm{Q} / \mathrm{E}] \mathrm{PW}[\mathrm{H} / \mathrm{Q} / \mathrm{R}] \mathrm{F}[\mathrm{F} / \mathrm{I} / \mathrm{L} / \mathrm{V}] \mathrm{V}[\mathrm{A} / \mathrm{I} / \mathrm{V}]$ and $\mathrm{D}[\mathrm{A} / \mathrm{S} / \mathrm{T}] \mathrm{xP}[\mathrm{I} / \mathrm{M}] \mathrm{EG}[\mathrm{F} / \mathrm{I} / \mathrm{V}][\mathrm{D} / \mathrm{H} / \mathrm{Q}])$. None of these conserved sequences are present in MsuE.

Figure 1. Nitroreductase-dependent activation of the fluorogenic probe FSL 61. E. coli SOS-R2 strains over-expressing NfsA_Ec, NfsB_Ec or MsuE as indicated, or containing an empty pUCX plasmid ("Control"), were streaked on an LB agar plate amended with $100 \mu \mathrm{g} / \mathrm{mL}$ ampicillin and $25 \mu \mathrm{M}$ of the nitro-quenched fluorophore FSL 61 (structure inset). The image was taken using a hand-held Panasonic DMC-LS5 digital camera, with the agar plate underlit by a broad-range ultra-violet transilluminator.

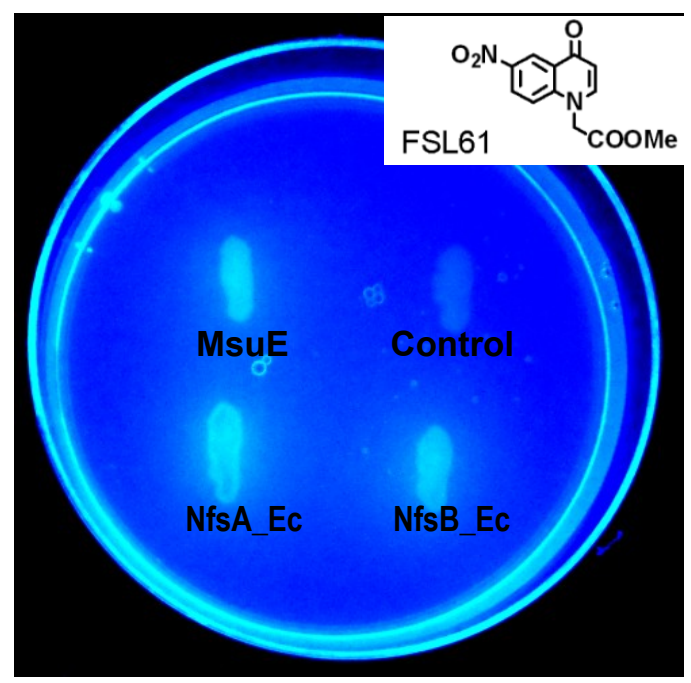

\subsection{MsuE Is Able to Activate Promising Next-Generation GDEPT Prodrugs}

We next conducted a preliminary test of whether MsuE was able to catalyse bioreductive activation of the prodrugs CB1954, PR-104A or nitro-CBI-DEI at comparable levels to the prototypical nitroreductases NfsA_Ec and NfsB_Ec. To achieve this, each enzyme was individually over-expressed in the E. coli reporter strain SOS-R2, which lacks endogenous nitroreductases and contains a lacZ gene under control of the SOS (DNA damage responsive) promoter sfiA. We have previously demonstrated that this reporter system provides a quantitative measure of genotoxic prodrug activation by over-expressed nitroreductase candidates [14]. Upon challenge with $20 \mu \mathrm{M}$ CB1954, only NfsA_Ec and NfsB_Ec induced a significant SOS response relative to the empty-plasmid control $(p<0.005$; Figure $3 \mathrm{~A})$. However, following challenge with $5 \mu \mathrm{M}$ nitro-CBI-DEI or $40 \mu \mathrm{M}$ PR-104A, all three enzymes generated a significant SOS response relative to the control ( $p<0.05$; Figure 3B,C). With nitro-CBI-DEI, all three enzymes had comparable activity in this system; while with PR-104A, MsuE was more active than NfsB_Ec, but less active than NfsA_Ec. 
Figure 2. Phylogenetic analysis of $\mathrm{MsuE}$ and other experimentally validated bacterial nitroreductase families. MsuE (dark red) and two SsuE orthologues from E. coli and Pseudomonas syringae pv. tomato DC3000 (orange) were genetically aligned against members of the NfsA (red), NfsB (dark blue), MdaB (black), YieF (black), NemA (pink), YwrO (light blue) and AzoR (green) nitroreductase families, and a human NQO1 out-group (grey), using the online tool ClustalW2. With the exception of the $m s u E$ and $s s u E$ genes, nitroreductase sequences are as previously presented by Prosser et al. [14]. Following the nomenclature of that paper, the two letter suffix following the enzyme name indicates the bacterial strain of origin: Ba, B. amyloliquefaciens; Bs, Bacillus subtilis; Ck, Citrobacter koseri; Es, Enterobacter sakazakii; Ec, Escherichia coli; Kp, Klebsiella pneumonia; Li, Listeria innocua; Pa, Pseudomonas aeruginosa; Pp, Pseudomonas putida; Ps, Pseudomonas syringae; St, Salmonella typhi; Vf, Vibrio fischeri; Vh, Vibrio harveyi; Vv, Vibrio vulnificus. The phylogenetic tree was constructed using the online tool MrBayes 3.2.1 [27] and bootstrap confidence values are indicated at major branchpoints. The final figure was generated using Geneious version 6.1 [28].

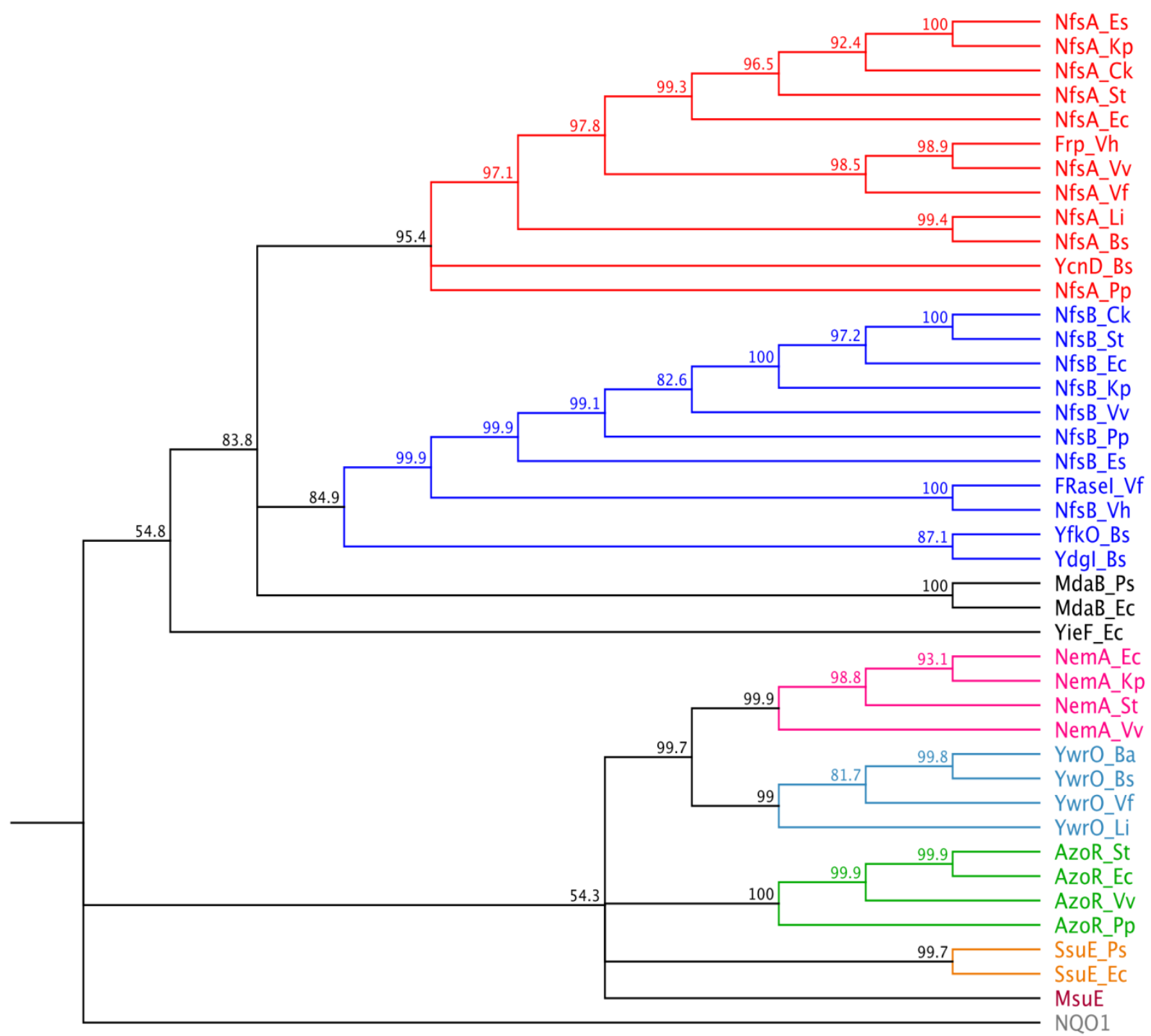


Figure 3. Fold-induction of SOS response in SOS-R2 strains over-expressing NfsA_Ec, NfsB_Ec or MsuE upon challenge with CB1954, nitro-CBI-DEI or PR-104A. SOS-R2 reporter strains over-expressing $n f s A_{-} E c, n f s B_{-} E c$ or $m s u E$, or containing an empty pUCX plasmid control, were incubated for $3 \mathrm{~h}$ in the presence of (A) $20 \mu \mathrm{M} \mathrm{CB} 1954$; (B) $5 \mu \mathrm{M}$ nitro-CBI-DEI; or (C) $40 \mu \mathrm{M}$ PR-104A (structures inset); after which relative induction of the SOS response in each strain was measured by $\beta$-galactosidase assay as previously described [13]. Data are the mean Miller units from three independent experiments, each performed in duplicate; and error bars are \pm 1 standard deviation. The black dotted line indicates the upper error limit of basal SOS activity in the empty plasmid control. * indicates $p<0.05, * *$ indicates $p<0.005, * * *$ indicates $p<0.001$ (one-way ANOVA with Dunnett comparison of test to control).

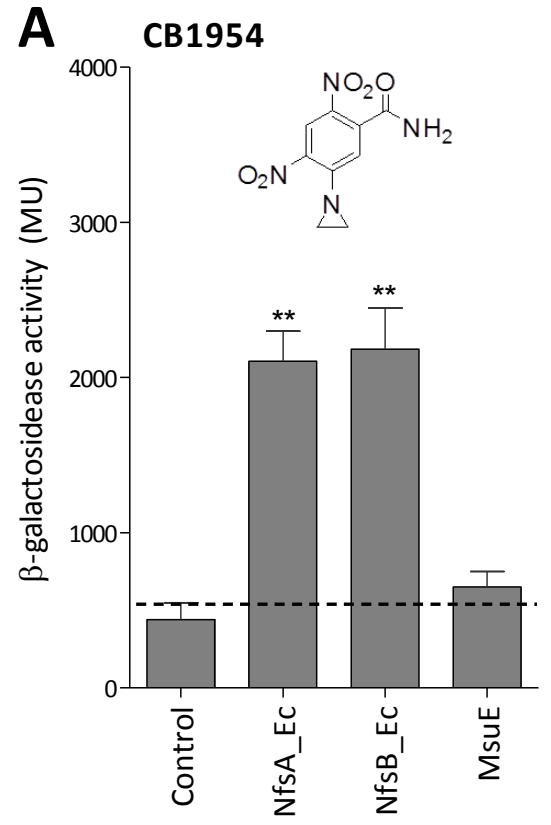

Over-expressed nitroreductase

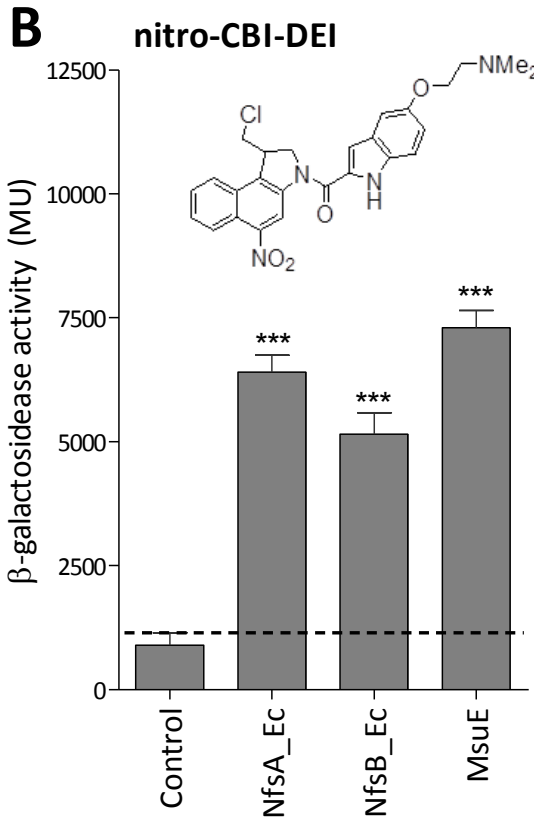

Over-expressed nitroreductase

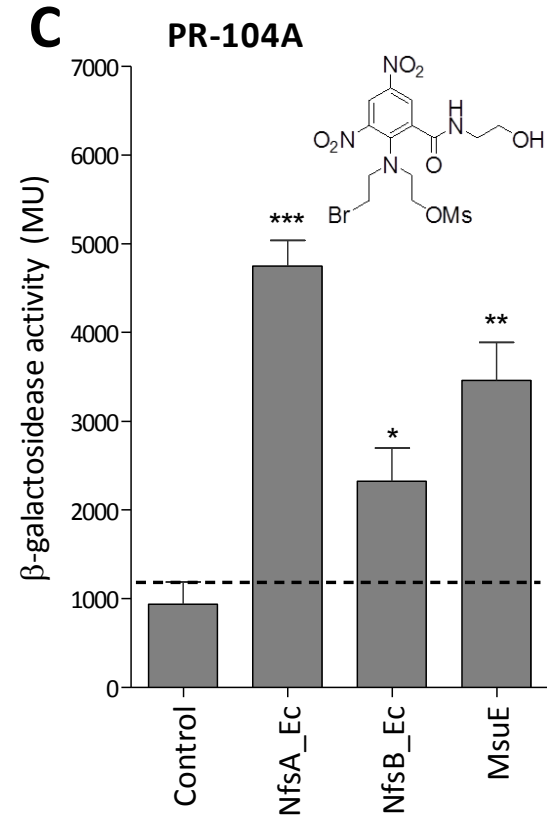

Over-expressed nitroreductase

\subsection{MsuE Is Able to Sensitise Human Colon Carcinoma Cells to Bioreductive Prodrugs}

In an effort to measure the relative abilities of MsuE, NfsA_Ec and NfsB_Ec to sensitise human colon carcinoma (HCT-116) cells to each prodrug we employed a bacteria-delivered enzyme cytotoxicity assay as previously described [13]. E. coli strains individually over-expressing each enzyme or an empty plasmid control were incubated in co-culture with replicate monolayers of HCT-116 cells, across a range of concentrations of each prodrug, after which $\mathrm{IC}_{50}$ values were measured (i.e., the concentration of prodrug required to reduce the viability of the HCT-116 cells to $50 \%$ relative to the no-prodrug control). In this assay system, independent experiments using CB1954 or nitro-CBI-DEI gave highly reproducible results for all strains. Despite MsuE exhibiting only limited activity with CB1954 in SOS assays (Section 2.3), HCT-116 cells co-cultured with the E. coli strain over-expressing this nitroreductase were 5.8-fold more sensitive to CB1954 than cells co-cultured with the empty plasmid control strain (Figure 4A). Consistent with the SOS assays, NfsA_Ec and NfsB_Ec were more effective than MsuE in sensitising HCT-116 cells to CB1954 (Figure 4A). With nitro-CBI-DEI however, MsuE 
was the most effective nitroreductase in sensitising HCT-116 cells to the administered prodrug (Figure 4B). The $\mathrm{IC}_{50}$ of $260 \pm 40 \mathrm{nM}$ measured in this assay system is comparable to that previously described for the most active nitro-CBI-DEI reductase yet to be identified ( $P$. aeruginosa NfsB, $130 \mathrm{nM}$ [17]); and substantially (although not quite significantly; $p<0.06$ ) less than that measured for the best $E$. coli nitroreductase, NfsA_Ec $(1.9 \pm 1.2 \mu \mathrm{M}$; Figure 4B). Surprisingly, PR-104A was unable to exert a comparable effect in this assay, possibly due to the relatively reactive nature of the metabolites; irrespective of the conditions employed, concentration-dependent $\mathrm{IC}_{50}$ curves could not be obtained for any of the E. coli strains. Overall, based on the results of the SOS and bacteria-delivered enzyme cytotoxicity assays, MsuE appears to be a promising candidate for GDEPT, offering potential to repurpose next-generation prodrugs that have been independently developed as hypoxia activated therapeutics.

Figure 4. Bacteria-delivered enzyme prodrug cytotoxicity assay. Calculated $\mathrm{IC}_{50}$ of HCT-116 cells post-incubation with E. coli $6 K O$ cells (over-expressing either NfsA_Ec, NfsB_Ec, MsuE, or an empty pUCX control) across a 2-fold dilution series of (A) CB1954 (25 $\mu \mathrm{M}$ to $400 \mu \mathrm{M})$; or (B) nitro-CBI-DEI $(0.06 \mu \mathrm{M}$ to $15 \mu \mathrm{M})$. Percentage cell survival at each prodrug concentration was calculated relative to an unchallenged control by CellTiter $96^{\circledR} \mathrm{AQ}_{\text {ueous }}$ One Solution Cell Proliferation Assay (Promega, Madison, WI, USA). Data are the mean of three independent experiments, each performed in duplicate; and error bars are \pm 1 standard deviation. $* *$ indicates $p<0.005$ and $* * * p<0.001$ by one-way ANOVA with Dunnett comparison of test to control.

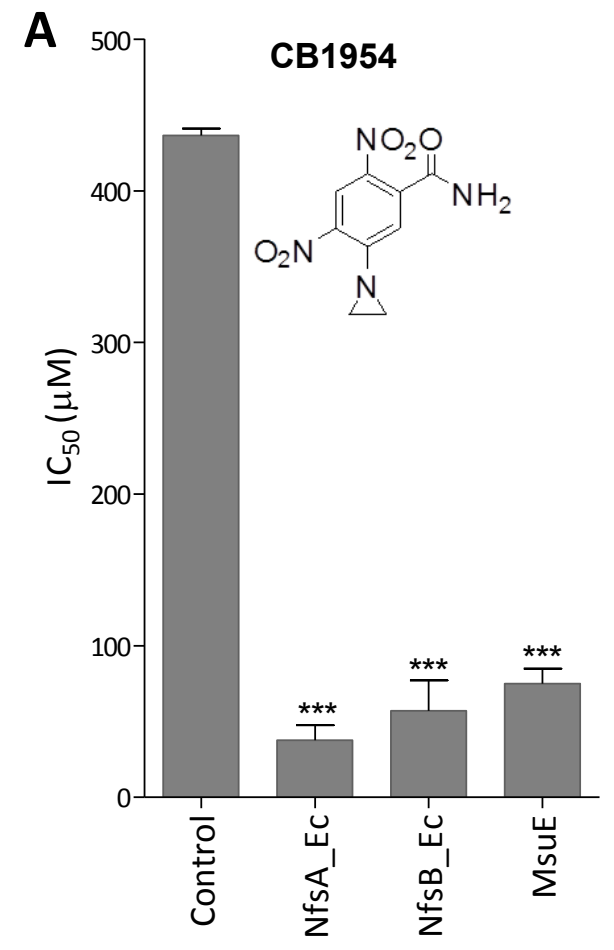

Over-expressed nitroreductase

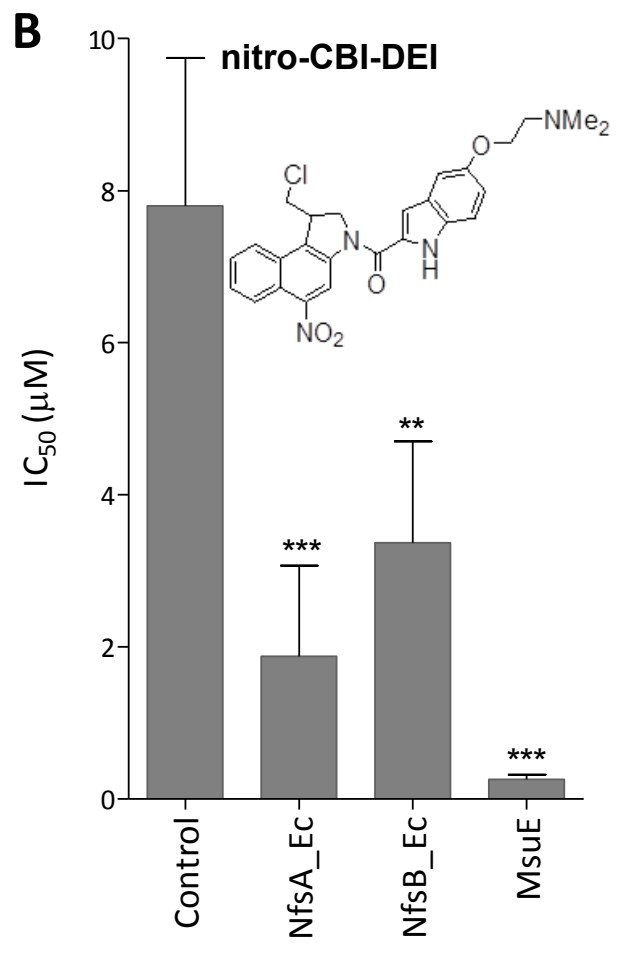

Over-expressed nitroreductase 


\section{Experimental}

\subsection{Chemicals, Bacterial Strains, Plasmids and Bacterial Culture Conditions}

Chemicals (including CB1954), reagents and growth media were obtained from Sigma-Aldrich (St. Louis, MO, USA) unless otherwise stated. PR-104A and the fluorogenic probe FSL61 were synthesised in-house at the Auckland Cancer Society Research Centre (ACSRC). Nitro-CBI-DEI was a generous gift from Dr Moana Tercel at the ACSRC.

The $E$. coli SOS reporter strain SOS-R2, gene deleted in four endogenous nitroreductases ( $n f_{S} A n f_{S} B$ $n e m A a z o R$ ) and the efflux regulator tolC (construction described in [14]) was used as the default host strain for all experiments involving nitroreductase gene over-expression unless otherwise stated. NfsA_Ec and NfsB_Ec were over-expressed from plasmid pUCX, an expression plasmid derived from pUC19, as previously described [11]. The $m s u E$ gene was PCR-amplified from P. aeruginosa PAO1 genomic DNA using oligonucleotide primers purchased from Integrated DNA Technologies (Coralville, IA, USA): $m s u E \_$for CCCCATATGACCAGCCCCTTCAAA and $m s u E \_r e v$ CCCGTCGACTCAGGCG ATCTTCAACGG; and cloned into pUCX using the NdeI and SalI restriction sites (underlined). Successful error-free PCR amplification and cloning was verified by sequencing (Macrogen Inc., Seoul, Korea). For bacteria-delivered enzyme prodrug cytotoxicity assays, all nitroreductases were expressed from pUCX in an $n f S A n f_{S} B$ azoR mdaB yieF ycaK (" $\Delta 6 K O$ ") deletion strain of E. coli W3110 (the intact tolC gene in this strain promotes efflux of activated metabolites relative to SOS-R2). Luria-Bertani (LB) liquid or solid (1.5\% agar) media containing $100 \mu \mathrm{g} / \mathrm{mL}$ ampicillin was used for propagation of $E$. coli strains harboring pUCX plasmids. Solid LB media was amended with $25 \mu \mathrm{M}$ FSL 61 for fluorescence screening.

\subsection{Bioinformatic Analyses}

The MsuE, SsuE_Ec and SsuE_Ps protein and gene sequences were downloaded from PubMed databases [29] (GenBank protein accession numbers AAG05745.1, BAA35692.1 and AAO56926.1, respectively). The multiple sequence alignment was conducted using ClustalW2 [30]. The phylogenetic tree in Figure 2 was reconstructed from this alignment using MrBayes 3.2.1 [27]. All other sequence analyses were performed using Geneious version 6.1 [28].

\subsection{SOS Assays}

Individual wells of a 96-well microtitre plate containing $100 \mu \mathrm{L}$ LB amended with $100 \mu \mathrm{g} / \mathrm{mL}$ ampicillin and $0.4 \%(\mathrm{w} / \mathrm{v})$ glucose were inoculated in duplicate with SOS-R2 strains over-expressing NfsA_Ec, NfsB_Ec or MsuE, or else containing a pUCX empty plasmid control, and incubated overnight at $30{ }^{\circ} \mathrm{C}$ with shaking at $200 \mathrm{rpm}$. The next morning, $15 \mu \mathrm{L}$ from each well was sub-cultured into $200 \mu \mathrm{L}$ of fresh medium (LB supplemented $100 \mu \mathrm{g} / \mathrm{mL}$ ampicillin, $0.42 \%(\mathrm{w} / \mathrm{v})$ glucose and $50 \mu \mathrm{M}$ IPTG) in a fresh 96-well microtitre plate. The fresh plates were then incubated at $30{ }^{\circ} \mathrm{C}$ with shaking at $200 \mathrm{rpm}$ for $3.5 \mathrm{~h}$ for induction of nitroreductase expression. Cultures were then split (100 $\mu \mathrm{L}$ each) into two fresh 96 well microtitre plates. Prodrug challenge was initiated in one of the replicate plates by addition of $100 \mu \mathrm{L}$ fresh assay media containing prodrug at $2 \times$ the desired final concentration and a 
DMSO concentration of $1 \%(\mathrm{v} / \mathrm{v})$. The remaining microtitre plate (unchallenged control) was treated identically except that prodrug was omitted from the media. After $3 \mathrm{~h}$ incubation at $30{ }^{\circ} \mathrm{C}$ with shaking (200 rpm), turbidity $\left(\mathrm{OD}_{600}\right)$ was recorded and then $10 \mu \mathrm{L}$ of each culture was added to $140 \mu \mathrm{L} Z \mathrm{OB}$ buffer (see below) for analysis of $\beta$-galactosidase activity in fresh 96-well microtitre plates. Reactions were then incubated at $37^{\circ} \mathrm{C}$ for $25 \mathrm{~min}$, at which point the assay was terminated by addition of $50 \mu \mathrm{L}$ of $1 \mathrm{M} \mathrm{Na}_{2} \mathrm{CO}_{3}$ and absorbance at $420 \mathrm{~nm}$ and $550 \mathrm{~nm}$ were recorded. Miller units of ONPG were calculated using a modified Miller equation as previously described [11]. The ZOB buffer used in this study was a 9:4:1 (v/v) mixture of $50 \mathrm{mM}$ sodium phosphate buffer ( $\mathrm{pH} 7.0), \mathrm{Z}$ buffer and $\mathrm{T}$ base, respectively, where $\mathrm{Z}$ buffer is $74 \mathrm{mM} \mathrm{NaH}_{2} \mathrm{PO}_{4}, 126 \mathrm{mM} \mathrm{Na}_{2} \mathrm{HPO}_{4}, 2 \mathrm{mM} \mathrm{MgSO}$, $0.4 \mathrm{mM} \mathrm{MnSO}_{4}$, $400 \mathrm{mg} / \mathrm{L}$ hexadecyltrimethylammonium bromide (CTAB), $200 \mathrm{mg} / \mathrm{L}$ sodium deoxycholate and $174 \mathrm{mM}$ $(12.2 \mu \mathrm{L} / \mathrm{mL}$ ) $\beta$-mercaptoethanol (the latter added immediately prior to use); and $\mathrm{T}$ base is $80 \mathrm{mM}$ $\mathrm{K}_{2} \mathrm{HPO}_{4}, 44 \mathrm{mM} \mathrm{KH} \mathrm{PO}_{4}, 1 \mathrm{~g} / \mathrm{L}$ trisodium citrate, $15.1 \mathrm{mM}\left(\mathrm{NH}_{4}\right)_{2} \mathrm{SO}_{4}$, and $8 \mathrm{~g} / \mathrm{L} \mathrm{ONPG}$ (the latter added immediately prior to use).

\subsection{Bacteria-Delivered Enzyme Prodrug Cytotoxicity Assays}

HCT-116 colorectal carcinoma cells (American Type Culture Collection, Manassas, VA, USA) were seeded into 96 -well microtitre plate wells at $5 \times 10^{3}$ per $100 \mu \mathrm{L}$ of cell culture media (CMEM supplemented with 5\% foetal calf serum and $10 \mathrm{mM}$ HEPES (Gibco, Grand Island, NY, USA) and incubated at $37{ }^{\circ} \mathrm{C}, 5 \% \mathrm{CO}_{2}$ for $48 \mathrm{~h}$. In separate 96-well microtitre plates, E. coli $\triangle 6 \mathrm{KO}$ strains over-expressing each nitroreductase from plasmid pUCX, or else transformed with an empty pUCX control, were individually inoculated into $150 \mu \mathrm{L} \mathrm{LB}$ amended with $100 \mu \mathrm{g} / \mathrm{mL}$ ampicillin and $0.4 \%(\mathrm{w} / \mathrm{v}$ ) glucose, then incubated for $16 \mathrm{~h}$ at $30{ }^{\circ} \mathrm{C}$ with shaking at $200 \mathrm{rpm}$. The next day, $15 \mu \mathrm{L}$ from each overnight culture were transferred to fresh 96 -well microtitre plate wells containing $200 \mu \mathrm{L}$ cell culture media amended with $100 \mu \mathrm{g} / \mathrm{mL}$ ampicillin, $0.05 \mathrm{mM} \mathrm{IPTG}$ and $0.4 \%$ (w/v) glucose (CMEM-AIG), and incubated at $30{ }^{\circ} \mathrm{C}$ with shaking at $200 \mathrm{rpm}$ for $3 \mathrm{~h}$. During this incubation period, a 2-fold dilution series of CB1954 (from $400 \mu \mathrm{M}$ to $25 \mu \mathrm{M}$, plus no-prodrug control) and nitro-CBI-DEI (from $15 \mu \mathrm{M}$ to $0.06 \mu \mathrm{M}$, plus no-prodrug control) were prepared in CMEM-AIG. The $3 \mathrm{~h}$ cultures of the bacterial over-expression strains were then assessed for uniform growth by $\mathrm{OD}_{600}$ measurement (standard error was $\pm 2.5 \%$ ) and mixed $1: 1$ with the prodrug dilution series in a final volume of $210 \mu \mathrm{L}$ per well. HCT-116 monolayers were then washed twice with $\mathrm{PBS}$, and $100 \mu \mathrm{L}$ of the bacteria-prodrug mixtures were applied. The monolayers were incubated $4 \mathrm{~h}$ at $37{ }^{\circ} \mathrm{C}, 5 \% \mathrm{CO}_{2}$, after which the bacteria were removed by washing four times in PBS and fresh $100 \mu \mathrm{L}$ DMEM (supplemented with foetal calf serum, HEPES and $100 \mu \mathrm{g} / \mathrm{mL}$ chloramphenicol to kill the bacteria) was added to each monolayer. The HCT-116 cells were then allowed to recover at $37{ }^{\circ} \mathrm{C}, 5 \% \mathrm{CO}_{2}$ for $48 \mathrm{~h}$. Following this period, $20 \mu \mathrm{L}$ of CellTiter $96^{\circledR}$ AQueous One Solution (Promega, Madison, WI, USA) was added to each well and incubated for $2 \mathrm{~h}$ at $37{ }^{\circ} \mathrm{C}, 5 \% \mathrm{CO}_{2}$. The $\mathrm{OD}_{490}$ was recorded to measure formazan levels, proportional to the number of respiring HCT-116 cells present in the culture. $\mathrm{IC}_{50}$ values were then calculated by comparing the $\mathrm{OD}_{490}$ of the challenged cells with the unchallenged cells using GraphPad Prism (Graph-pad software Inc., La Jolla, CA, USA). 


\section{Conclusions}

Historically, based on their original isolation from partially purified E. coli extracts that contained different nitrofurazone reducing activities, NfsA has been referred to as the "major" oxygen-insensitive nitroreductase and NfsB the "minor" nitroreductase [31-33]. These designations, however, do not do justice to the wealth of other enzymes (represented in E. coli as well as other bacteria) that possess nitro-reducing capabilities; nor to the fact that NfsB exhibits comparable activity to NfsA with a number of different nitroaromatic substrates [12,34], both enzymes typically being far more active than representatives of families such as NemA, AzoR and MdaB [14]. MsuE is the first representative outside of the NfsA and NfsB families to display comparable activity with next-generation GDEPT prodrugs. This is of particular interest to us given our ongoing interest in both rationally engineering and randomly evolving superior nitroreductase variants from wild type progenitors [13]; the structural and functional novelty that an entirely new nitroreductase offers may enable unique solutions to problems of achieving superior catalytic activity. On this basis, we suggest that the results presented here merit further evaluation of MsuE in in vivo GDEPT models, especially in partnership with nitro-CBI-DEI prodrug.

\section{Acknowledgments}

This research was supported by the Health Research Council of New Zealand (project contract 06/229 to DFA and programme contract 11/1103 to AVP, JBS and DFA), the Royal Society of New Zealand Marsden Fund (contract VUW0704 to DFA, AVP and JBS), and the New Zealand Lottery Health Research Fund (contract 313732 to DFA and JNC). We thank Moana Tercel for her generous gift of nitro-CBI-DEI prodrug.

\section{Conflict of Interest}

The authors declare no conflict of interest.

\section{References}

1. Dachs, G.U.; Hunt, M.A.; Syddall, S.; Singleton, D.C.; Patterson, A.V. Bystander or no bystander for gene directed enzyme prodrug therapy. Molecules 2009, 14, 4517-4545.

2. Portsmouth, D.; Hlavaty, J.; Renner, M. Suicide genes for cancer therapy. Mol. Aspects Med. 2007, 28, 4-41.

3. Rainov, N.G. A phase III clinical evaluation of HSV thymidine kinase and ganciclovir gene therapy as an adjuvant to surgical resection and radiation in adults with previously untreated glioblastoma multiforme. Hum. Gene Ther. 2000, 11, 2389-2401.

4. Freytag, S.O.; Khil, M.; Stricker, H.; Peabody, J.; Menon, M.; DePeralta-Venturina, M.; Nafziger, D.; Pegg, J.; Paielli, D.; Brown, S.; et al. Phase I study of replication-competent adenovirus-mediated double suicide gene therapy for the treatment of locally recurrent prostate cancer. Cancer Res. 2002, 62, 4968-4976.

5. Tanaka, T.; Duflot-Dancer, A.; Tiraby, M.; Piccoli, C.; Tiraby, G.; Yamasaki, H.; Mesnil, M. Bystander effect from cytosine deaminase and uracil phosphoribosyl transferase genes in vitro: A partial contribution of gap junctions. Cancer Lett. 2009, 282, 43-47. 
6. Chung-Faye, G.; Palmer, D.; Anderson, D.; Clark, J.; Downes, M.; Baddeley, J.; Hussain, S.; Murray, P.I.; Searle, P.; Seymour, L.; et al. Virus-directed, enzyme prodrug therapy with nitroimidazole reductase: A phase I and pharmacokinetic study of its prodrug, CB1954. Clin. Cancer Res. 2001, 7, 2662-2668.

7. Patel, P.; Young, J.G.; Mautner, V.; Ashdown, D.; Bonney, S.; Pineda, R.G.; Collins, S.I.; Searle, P.F.; Hull, D.; Peers, E.; et al. A phase I/II clinical trial in localized prostate cancer of an adenovirus expressing nitroreductase with CB1954 [correction of CB1984]. Mol. Ther. 2009, 17, 1292-1299. Erratum in: Mol. Ther. 2009, 17, 1302.

8. Knox, R.J.; Friedlos, F.; Jarman, M.; Roberts, J.J. A new cytotoxic, DNA interstrand crosslinking agent, 5-(aziridin-1-yl)-4-hydroxylamino-2-nitrobenzamide, is formed from 5-(aziridin-1-yl)-2,4dinitrobenzamide (CB 1954) by a nitroreductase enzyme in Walker carcinoma cells. Biochem. Pharmacol. 1988, 37, 4661-4669.

9. Anlezark, G.M.; Melton, R.G.; Sherwood, R.F.; Coles, B.; Friedlos, F.; Knox, R.J. The bioactivation of 5-(aziridin-1-yl)-2,4-dinitrobenzamide (CB1954) - I. Purification and properties of a nitroreductase enzyme from Escherichia coli-A potential enzyme for antibody-directed enzyme prodrug therapy (ADEPT). Biochem. Pharmacol. 1992, 44, 2289-2295.

10. Onion, D.; Patel, P.; Pineda, R.G.; James, N.; Mautner, V. Antivector and tumor immune responses following adenovirus-directed enzyme prodrug therapy for the treatment of prostate cancer. Hum. Gene Ther. 2009, 20, 1249-1258.

11. Prosser, G.A.; Copp, J.N.; Syddall, S.P.; Williams, E.M.; Smaill, J.B.; Wilson, W.R.; Patterson, A.V.; Ackerley, D.F. Discovery and evaluation of Escherichia coli nitroreductases that activate the anti-cancer prodrug CB1954. Biochem. Pharmacol. 2010, 79, 678-687.

12. Prosser, G.A.; Patterson, A.V.; Ackerley, D.F. uvrB gene deletion enhances SOS chromotest sensitivity for nitroreductases that preferentially generate the 4-hydroxylamine metabolite of the anti-cancer prodrug CB1954. J. Biotechnol. 2010, 150, 190-194.

13. Swe, P.M.; Copp, J.N.; Green, L.K.; Guise, C.P.; Mowday, A.M.; Smaill, J.B.; Patterson, A.V.; Ackerley, D.F. Targeted mutagenesis of the Vibrio fischeri flavin reductase FRase I to improve activation of the anticancer prodrug CB1954. Biochem. Pharmacol. 2012, 84, 775-783.

14. Prosser, G.A.; Copp, J.N.; Mowday, A.M.; Guise, C.P.; Syddall, S.P.; Williams, E.M.; Horvat, C.N.; Swe, P.M.; Ashoorzadeh, A.; Denny, W.A.; et al. Creation and screening of a multi-family bacterial oxidoreductase library to discover novel nitroreductases that efficiently activate the bioreductive prodrugs CB1954 and PR-104A. Biochem. Pharmacol. 2013, 85, 1091-1103.

15. Wilson, W.R.; Pullen, S.M.; Hogg, A.; Helsby, N.A.; Hicks, K.O.; Denny, W.A. Quantitation of bystander effects in nitroreductase suicide gene therapy using three-dimensional cell cultures. Cancer Res. 2002, 62, 1425-1432.

16. Wilson, W.R.; Stribbling, S.M.; Pruijn, F.B.; Syddall, S.P.; Patterson, A.V.; Liyanage, H.D.S.; Smith, E.; Botting, K.J.; Tercel, M. Nitro-chloromethylbenzindolines: Hypoxia-activated prodrugs of potent adenine N3 DNA minor groove alkylators. Mol. Cancer Ther. 2009, 8, 2903-2913.

17. Green, L.K.; Syddall, S.P.; Carlin, K.M.; Bell, G.D.; Guise, C.P.; Mowday, A.M.; Hay, M.P.; Smaill, J.B.; Patterson, A.V.; Ackerley, D.F. Pseudomonas aeruginosa NfsB and nitro-CBI-DEIA promising enzyme/prodrug combination for GDEPT. Mol. Cancer 2013, 12, 58. 
18. Patterson, A.V.; Ferry, D.M.; Edmunds, S.J.; Gu, Y.; Singleton, R.S.; Patel, K.; Pullen, S.M.; Hicks, K.O.; Syddall, S.P.; Atwell, G.J.; et al. Mechanism of action and preclinical antitumor activity of the novel hypoxia-activated DNA cross-linking agent PR-104. Clin. Cancer Res. 2007, 13, 3922-3932.

19. Denny, W.A. Prospects for hypoxia-activated anticancer drugs. Curr. Med. Chem. Anticancer Agents 2004, 4, 395-399.

20. Barak, Y.; Thorne, S.H.; Ackerley, D.F.; Lynch, S.V.; Contag, C.H.; Matin, A. New enzyme for reductive cancer chemotherapy, YieF, and its improvement by directed evolution. Mol. Cancer Ther. 2006, 5, 97-103.

21. Theys, J.; Pennington, O.; Dubois, L.; Anlezark, G.; Vaughan, T.; Mengesha, A.; Landuyt, W.; Anné, J.; Burke, P.J.; Dûrre, P.; et al. Repeated cycles of Clostridium-directed enzyme prodrug therapy result in sustained antitumour effects in vivo. Br. J. Cancer 2006, 95, 1212-1219.

22. Copp, J.N.; Williams, E.W.; Ackerley, D.F. Victoria University, Wellington, New Zealand. Unpublished work, 2013.

23. Roldán, M.D.; Pérez-Reinado, E.; Castillo, F.; Moreno-Vivián, C. Reduction of polynitroaromatic compounds: The bacterial nitroreductases. FEMS Microbiol. Rev. 2008, 32, 474-500.

24. Kertesz, M.A.; Schmidt-Larbig, K.; Wüest, T. A novel reduced flavin mononucleotide-dependent methanesulfonate sulfonatase encoded by the sulfur-regulated msu operon of Pseudomonas aeruginosa. J. Bacteriol. 1999, 181, 1464-1473.

25. Robinson, C.V.; Elkins, M.R.; Bialkowski, K.M.; Thornton, D.J.; Kertesz, M.A. Desulfurization of mucin by Pseudomonas aeruginosa: Influence of sulfate in the lungs of cystic fibrosis patients. J. Med. Microbiol. 2012, 61, 1644-1653.

26. Ernster, L.; Navazio, R. Soluble diaphorase in animal tissues. Acta Chem. Scand. 1958, 12, 595-602.

27. MrBayes. Available online: http://mrbayes.sourceforge.net/ (accessed on 23 July 2013).

28. Geneious, version 6.1; Biomatters: San Francisco, CA, USA, 2013.

29. NCBI. Available online: http://www.ncbi.nlm.nih.gov/pubmed/ (accessed on 23 July 2013).

30. Clustalw2. Available online: http://www.ebi.ac.uk/Tools/msa/clustalw2/ (accessed on 23 July 2013).

31. Bryant, D.W.; McCalla, D.R.; Leeksma, M.; Laneuville, P. Type I nitroreductases of Escherichia coli. Can. J. Microbiol. 1981, 27, 81-86.

32. Zenno, S.; Koike, H.; Kumar, A.N.; Jayaraman, R.; Tanokura, M.; Saigo, K. Biochemical characterization of NfsA, the Escherichia coli major nitroreductase exhibiting a high amino acid sequence homology to Frp, a Vibrio harveyi flavin oxidoreductase. J. Bacteriol. 1996, 178, 4508-4514.

33. Zenno, S.; Koike, H.; Tanokura, M.; Saigo, K. Gene cloning, purification, and characterization of $\mathrm{NfsB}$, a minor oxygen-insensitive nitroreductase from Escherichia coli, similar in biochemical properties to FRase I, the major flavin reductase in Vibrio fischeri. J. Biochem. 1996, 120, 736-744. 
34. Vass, S.O.; Jarrom, D.; Wilson, W.R.; Hyde, E.I.; Searle, P.F. E. coli NfsA: An alternative nitroreductase for prodrug activation gene therapy in combination with CB1954. Br. J. Cancer 2009, 100, 1903-1911.

(C) 2013 by the authors; licensee MDPI, Basel, Switzerland. This article is an open access article distributed under the terms and conditions of the Creative Commons Attribution license (http://creativecommons.org/licenses/by/3.0/). 